\title{
Computer-aided Diagnosis for Internal Hemorrhoids by Measuring the Congestive Extent in Endoscopic Images
}

\author{
Koji Abe ${ }^{1}$, Hidenori Takagi ${ }^{2}$, Masahide Minami ${ }^{3}$ and Haiyan Tian ${ }^{4}$ \\ ${ }^{1}$ Department of Informatics, School of Science and Engineering, Kinki University, Japan; \\ ${ }^{2}$ Interdisciplinary Graduate School of Science and Engineering, Kinki University, Japan; \\ ${ }^{3}$ Graduate School of Medicine, The University of Tokyo, Japan; \\ ${ }^{4}$ Graduate School of Engineering, Kobe University, Japan; \\ ${ }^{1}$ koji@info.kindai.ac.jp, ${ }^{2}$ tkghdnr2@gmail.co.jp, ${ }^{3}$ maminami@dream.com, ${ }^{4}$ tian.haiyan2006@gmail.com
}

\begin{abstract}
This paper presents a system of computer-aided diagnosis for internal hemorrhoids based on the congestive extent using endoscopic images. This system could be effective for young or even general practitioners as a second opinion. In the proposed method, a pre-processing is conducted to the images for enhancing saturation and contrast of congestive regions and blood vessels. Next, considering characteristics of internal hemorrhoids, the proposed method measures degree of the congestion in the images, and extracts the congestive region and abnormalities on the congestive extent. Experimental results of the discrimination using the proposed abnormalities between normal and abnormal cases for 204 images including 108 abnormal cases have shown that the abnormalities are well effective to diagnose the congestion in the hemorrhoids.
\end{abstract}

Keywords: Computer-aided diagnosis; Internal hemorrhoids; Congestion; Medical image processing; Endoscopic image.

\section{Introduction}

Internal hemorrhoids are one of diseases in the anus. The hemorrhoids come from the congestion of the blood caused by sitting for long time at work, baby care, etc. With labor circumstances in the times, at least $70-80 \%$ of population in developed countries could potentially have symptoms of the hemorrhoids. However, since there is no nerve in the rectum, most of people hardly notice the symptoms and the hemorrhoids have been already progressing when we feel the symptoms. The early stage of the hemorrhoids is the congestion, and the symptom changes into appearance of projection and swellings, and then folds with the progression of the hemorrhoid. Therefore, the congestion is the most fundamental symptom in hemorrhoids.

When diagnosticians check internal hemorrhoids, they insert the endoscope from the anus into the rectum. Scenes of the rectum taken by the endoscope are shown to them via a monitor. At that time, if diagnosticians confirm a lesion of internal hemorrhoids, they save several endoscopic images of the lesion as a material for future observations. However, since it is difficult for even experts on hemorrhoids to diagnose internal hemorrhoids, disagreement between diagnosticians is often 
Koji Abe, Hidenori Takagi, Masahide Minami and Haiyan Tian; Computer-aided Diagnosis for Internal Hemorrhoids by Measuring the Congestive Extent in Endoscopic Images, Journal of Biomedical Engineering and Medical Imaging, Volume 1, No 6, Dec (2014) , pp 10-21

happened. For the reasons, a computer-aided diagnosis (CAD) system for evaluating internal hemorrhoids is required as a second opinion for diagnosticians. Measuring abnormality of the congestion in internal hemorrhoids could be useful for an assisted system for new doctors and nonexperts.

Diagnosticians judge the congestion in internal hemorrhoids based on only their experience after they observe the color and the size of the congestive part. Although there is no report on extracting abnormalities for the congestive region in medical images, as similar trials in the field of medical image processing, recognition of lesions [1, 2], segmentations of organs or objects such as bleeding regions [36], etc. have been reported. However, all of them are effective to only cases when contours of recognition parts are clear in images. Since the contour of the congestion is not clear in many cases due to shade around the contour, it would be difficult to apply them to the extraction of the congestion area. And, even if another technique to recognize an object in a gradation area like landscape images were existed, it would be hard to recognize the congestive region in endoscopic images because boundary between the region and its background is very dark in most cases due to shade, i.e., characteristics of color are hardly appeared around the boundary.

To design a CAD for internal hemorrhoids, this paper proposes a method for extracting the congestive extent of the hemorrhoids as abnormalities from endoscopic images. In addition, using the abnormalities, the proposed method diagnoses the images by discriminating between normal and abnormal cases as a prototype of the CAD. The abnormalities are measured by extracting characteristics of density distribution in the images. And then, this paper examines performance of the abnormalities from experimental results obtained by the discriminations.

\section{Endoscopic Images of Internal Hemorrhoids and the Congestion of the Blood}

Symptoms of internal hemorrhoids appeared in the endoscopic image are broadly divided into the following three: 1) the congestive region appears around the tube or its tip, 2) projections or swellings appear around the tube with the anal canal opened, and 3) the folds appear around the tube due to hard projections and swellings. Figure 1 shows an example of each case, where the leftmost is a normal case and the other three are abnormal cases. And, the black cylindrical object in every image shown in Figure 1 is the tube of the endoscope. In the normal case, the whole color of internal rectum looks pink and there is no significant difference of color between the whole color and the area around the tube. On the other hand, as shown in the image of "congestion" in Figure 1, we can see the color is changed into blackish purple around the tube. This part is the congestive region of the blood and a main factor to diagnose internal hemorrhoids caused by the congestion. Basically, the congestive region is taken around the tube in endoscopic images. When a diagnostician take a picture in the rectum using an endoscope, sometimes the tube is not appeared in the image depending on location of the taking place and camera lens. Considering the characteristic of the congestion in the image, only the images which include the tube region are used in this research. 


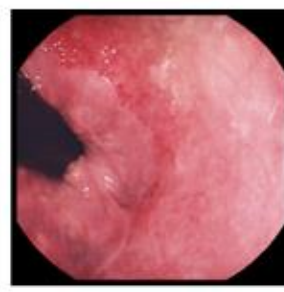

normal

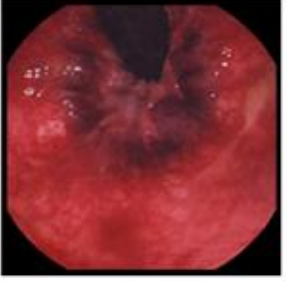

congestion

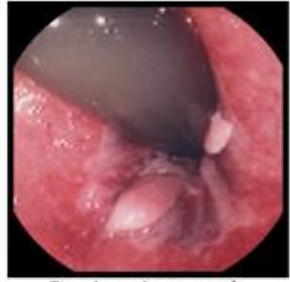

Projection and swellings

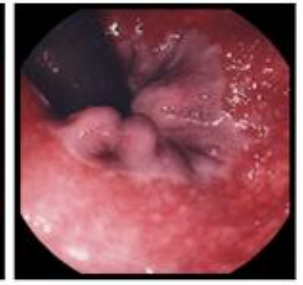

folds

Figure 1: Typical cases of internal hemorrhoids

To the endoscopic images, this paper proposes features for measuring the congestion extent such cases shown at "congestion" in Figure 1. Figure 2 shows difference of the histogram on each of red and blue values in RGB color space between a normal part and a congestive part, where the size of both of the two rectangles in the image is $40 \times 60$; and the histograms have been measured in each of the areas. In addition, Table 1 shows the mean values and the standard deviations for the histograms. From Figure 2 and Table 1, we can confirm that difference between red and blue values in the congestive part is much smaller than normal part, and red values in the congestive part are lower than normal part.

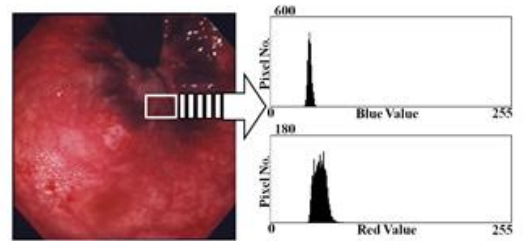

(a) congestion area

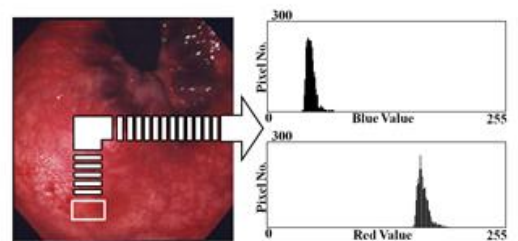

(b) normal area

Figure 2: Difference of color between the congestive region and normal area.

Table 3: The Mean value and the standard deviation of the histograms show in Fig. 2.

\begin{tabular}{|l|c|c|}
\hline & Mean value & Standard deviation \\
\hline red value in (a) & 55.4 & 32.7 \\
red value in (b) & 174.6 & 36.4 \\
\hline blue value in (a) & 44.0 & 57.3 \\
blue value in (b) & 48.8 & 41.2 \\
\hline
\end{tabular}

\section{Proposed Method}

Throughout this paper, the size of all the images is $512 \times 512$ pixels with 24 -bit full color.

\subsection{Preprocessing}

First, since the color of the tube region in the image is nonrelative in recognition of the congestive region and feature extractions of the congestive extent, and besides, there is possibility the region could be a noise due to much halation, the tube region is extracted from the original image by the Lazy Snapping (LS) [7] and all the pixel values in the tube region are converted into black color (i.e., the tube region is removed from the original image). The extraction is conducted according to the manual shown below. LS is a method for separating an image into the target area and its background by roughly drawing the boundary between them with an interface, hence the user manually cuts the target drawing 
Koji Abe, Hidenori Takagi, Masahide Minami and Haiyan Tian; Computer-aided Diagnosis for Internal Hemorrhoids by Measuring the Congestive Extent in Endoscopic Images, Journal of Biomedical Engineering and Medical Imaging, Volume 1, No 6, Dec (2014) , pp 10-21

a boundary between the two areas with a mouse or a tablet-pan on the monitor. Figure 3 shows a case when LS is applied to one of the endoscopic images, where (1) is the original image, (2) is a curve drawn in the inside of the tube region along the tube contour, (3) is a curve drawn in the outside of the tube region along the contour, and (4) is the final output. As shown in Figure 3(4), only the color of tube region is converted into black color. The manual and Figure 3 are shown to the user as a guide for drawing the curves.

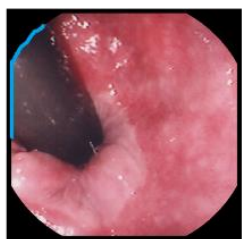

(1)

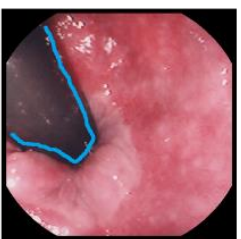

(2)

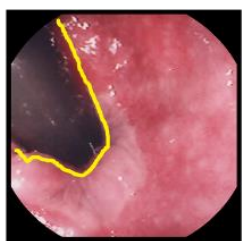

(3)

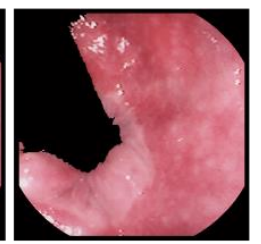

(4)

Figure 3: Extraction of the tube region by the Lazy Snapping.

\section{[Manual for extracting the tube region]}

1. Draw a blue curve on the tangential line of the tube region and the frame of the image.

$2^{*}$. Draw a blue curve inside of the tube region along the counter of the tube region.

$3^{*}$. Draw a yellow curve outside of the tube region along the counter of the tube region.

* Be careful not to draw the curves on the tube edge or the outside flame of the image.

Next, to the image conducted the tube removal, pixel values in RGB space are converted into HSV space and the linear stretch with saturation is conducted. In the stretch, the histogram for saturation value $S$ is stretched at the range $[0,1]$. After the stretch, pixel values are reconverted into values in RGB space. Figure 4 shows a case when the stretch is conducted, where the left is an image before the stretch and the right is the converted image. By this conversion, the congestion area and blood vessels could be brighter. RGB values are converted into HSV values $H$ (hue), $S$ (saturation), and $V$ (value) as follows:

$$
\begin{aligned}
& H=60 \times \begin{cases}\frac{G-B}{\max -\min } & \text { if max is } R \\
\frac{B-R}{\max -\min }+120 & \text { if max is } G \\
\frac{R-G}{\max -\min }+240 & \text { if } \max \text { is } B\end{cases} \\
& S=\frac{\max -\min }{\max } \\
& V=\max
\end{aligned}
$$

where $R, G$, and $B$ are color values of red, green, and blue in the pixel, respectively; and max and $\min$ are the highest and the lowest values among $R, G$, and $B$.

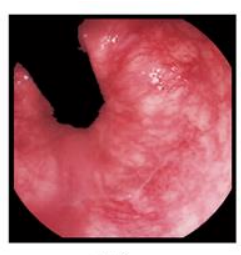

before

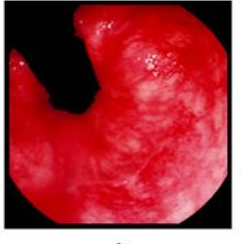

after

Figure 4: The linear stretch with saturation. 


\subsection{Extraction of an abnormality on Congestion: $f_{1}$}

As shown in Figure 2, red values of the blackish purple color appeared in the congestive region are basically higher than blue values. However, supposing features of the congestive extent were extracted considering only this characteristic, there could be possibility that halation and light-colored area become strong noises in the extraction. Besides, the red values are generally lower than red values in normal area. Considering them, an abnormality on congestion $f_{1}$ is given by

$$
\begin{gathered}
f_{1}=\sum_{x=0}^{512} \sum_{y=0}^{512} P(x, y) \\
P(x, y)=\frac{B(x, y)}{R(x, y)} \times w_{R}(x, y)
\end{gathered}
$$

Where $w_{R}(x, y)\left(0 \leq w_{R}(x, y) \leq 1\right)$ is the weight determined by red value $R$ and blue value $B$ in the pixel $(x$, $y)$. Thus, $f_{1}$ would be an abnormality considering the size of the congestive region and its color because $f_{1}$ measures the shade of the blackish purple with the ratio of $B$ for $R$ to all the pixels in the image.

\subsubsection{Derivation of the Weight $w_{R}(x, y)$}

The weight $w_{R}$ in the pixel $(x, y)$ is determined by employing the following sigmoid function:

$$
w_{R}(x, y)=\frac{1}{1+e^{-a(-127+R(x, y)-a x i s)}}
$$

Where $a$ is the gain value of the function, $R(x, y)$ is red value in the pixel $(x, y)$, and axis is defined as $x$ coordinate at the axis of symmetry in the sigmoid curve. The reason why the sigmoid function is employed is because the abnormality would be more effective in the discrimination between normal and abnormal cases if we could arrange difference of abnormality between normal and abnormal cases as much as possible around the boundary between groups of normal case and abnormal case. Therefore, the more $R(x, y)$ is small, the more $w_{R}$ is large (i.e., $w_{R}$ approaches 1 ), hence $f_{1}$ is going to the ratio of $\mathrm{B}$ for $\mathrm{R}$ shown in Eq.(5). On the other hand, the more $R(x, y)$ is large, the more $w_{R}$ is small (i.e., $w_{R}$ approaches 0 ), hence the ratio is revised low and $f_{1}$ is held down. As an example of the sigmoid function given by Eq.(6), Figure 5 shows a sigmoid curve for Eq.(6) where $a$ and axis are 0.5 and 0, respectively.

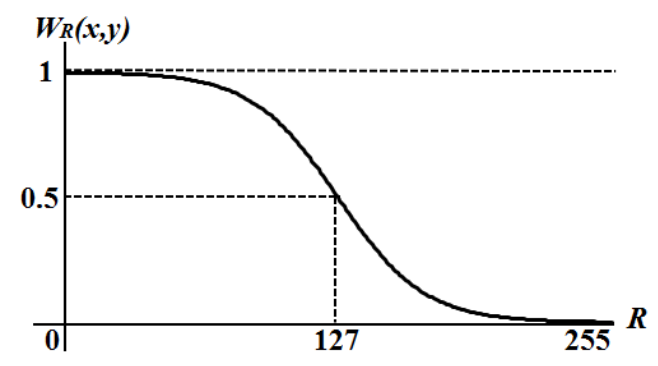

Figure 5: Sigmoid curve for Eq.(6) (a: 0.5, axis: 0) 


\subsubsection{Determination of the parameters $a$ and $a x i s$ in the weight $w_{R}(x, y)$}

The parameters a and axis are determined using samples. The samples should be chosen evenly from each dataset of normal and abnormal cases. In order to choose the samples evenly in the vector space, principal component analysis is applied to all the images in each case separately. The variables for the analysis are regarded as the three color values in RGB. By the analysis, the images are represented as vectors which have three coordinates of the first, second, and third principal components in a 3D space composed of the components. Putting all the images in each class in the 3D space, the image which is the nearest vector from the mean vector in each of the 8 quadrants in the 3D space is selected, i.e., 8 images are selected as the samples from each dataset of normal and abnormal cases (i.e., 16 images are totally selected.). Second, using $f_{1}$ extracted from the samples, degree of separation between the couple of datasets composed of the samples in each case is calculated. The degree of separation Sep is given by

$$
\begin{gathered}
\text { Sep }=\frac{\partial_{b}^{2}}{\partial_{w}^{2}} \\
\partial_{b}^{2}=\frac{N_{n} N_{a}\left(m_{n}-m_{a}\right)^{2}}{\left(N_{n}+N_{a}\right)^{2}} \\
\partial_{w}^{2}=\frac{N_{n} \partial_{n}^{2}+N_{a} \partial_{a}^{2}}{N_{n}+N_{a}}
\end{gathered}
$$

Where $N_{n}$ and $N_{a}$ are the number of the samples in each of normal and abnormal cases $\left(N_{n}=N_{a}=8\right), m_{n}$ and $m_{a}$ are the mean value of $f_{1}$ extracted from the samples in each, $\partial^{2}{ }_{n}$ and $\partial^{2}{ }_{a}$ are variance of $f_{1}$ in each, respectively. Third, changing both values of axis in the range [-127, 127] (pitch: 1) and $a$ in the range $[0.1,10.0]$ (pitch: 0.1), Sep is calculated each time. And then, the couple of $a$ and axis used in the case when Sep obtains the largest value is determined as their optimum values at last.

\subsection{Extraction of the Congestive Region}

The congestive region is extracted by the linear discriminant analysis with $P(x, y)$ shown in Eq.(5). The discriminant analysis is applied to every pixel in the image and their discriminant score decides whether the pixel belongs in the congestive region or its background. If the score for a pixel is more than 0 , the pixel belongs in the congestive region. Figure 6 shows the extracted congestive region for a case, where $(1)$ is the original image, (2) is the extracted congestive region.

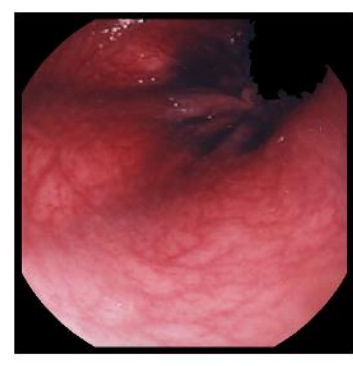

(1)

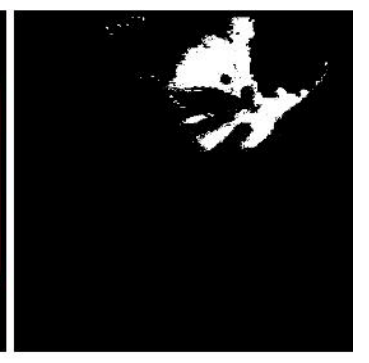

(2)

Figure 6: Extraction of the congestive region for a case. 


\subsection{Extraction of the abnormalities: $f 2, f 3$, and $f 4$}

In addition to $f_{1}$ proposed in $\mathbf{3 . 2}$, the other abnormalities on the congestive extent $f_{2}, f_{3}$ and $f_{4}$ are extracted as features from the extracted congestive region obtained in 3.3. $f_{2}$ represents the size of the congestive region. $f_{3}$ represents the mean value of red values in the congestive region. And, $f_{4}$ represents the mean value of the ratio of the blue values for the red values in the congestive region. Now, when the number of pixels in the congestive region is size and one of them is represented by $c_{i}(1$ $\leq i \leq$ size), the abnormalities are given by

$$
\begin{gathered}
f_{2}=\text { size } \\
f_{3}=\frac{1}{\text { size }} \sum_{i=1}^{\text {size }} r_{i} \\
f_{4}=\frac{1}{\text { size }} \sum_{i=1}^{\text {size }} \frac{b_{i}}{r_{i}}
\end{gathered}
$$

where $r_{i}$ and $b_{i}$ are the red and the blue values of the pixel $c_{i}$, respectively.

\section{Experimental Results}

To examine performance of the proposed abnormalities, linear discriminant analysis (LDA), neural network (NN), linear support vector machine (L-SVM), and nonlinear support vector machine (N-SVM) were applied to the discrimination of the endoscopic images into normal or abnormal (i.e., internal hemorrhoids) cases by regarding the abnormalities as variables, respectively. Each of the abnormalities was normalized to the dataset that the mean value is 0 and the variance is 1 . NN was designed as a three-layered perceptron (input layer: 3, hidden layer: 5, output layer: 3), where sigmoid function and back propagation (learning rate: 0.3 , weight decay rate: 0.1 ) were used. As a kernel function in N-SVM, the Gaussian kernel was used.

Selecting the samples for each of normal and abnormal cases from the dataset, the parameters $a$ and axis in Eq.(6) were obtained. Figure 7 shows Sep vs. the parameters $a$ and axis. From the result shown in Figure 7, the parameters $a$ and axis were obtained as 4.1 and -2 , respectively. The number of the images is 204 (normal case: 96, abnormal cases: 108), where the 16 samples selected to determine $a$ and axis were removed. In the experiments, each of three users $A, B$, and $C$, which are non-expert, drew the curves for the tube region seeing Figure 3 and the manual shown in 3.1, and the experiments were conducted to each of the users.

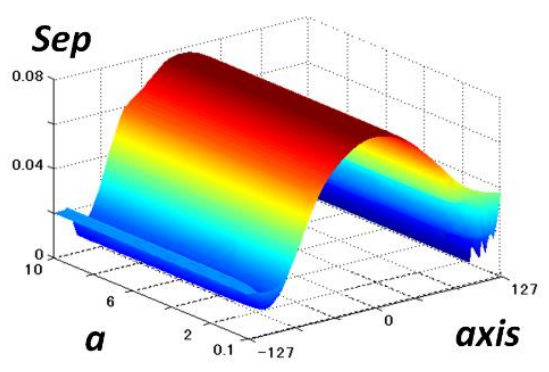

Figure 7: Sep vs. $a$ and axis. 
Koji Abe, Hidenori Takagi, Masahide Minami and Haiyan Tian; Computer-aided Diagnosis for Internal Hemorrhoids by Measuring the Congestive Extent in Endoscopic Images, Journal of Biomedical Engineering and Medical Imaging, Volume 1, No 6, Dec (2014) , pp 10-21

The training dataset and test dataset were chosen by the leave-one-out cross validation [8] in all the discriminations. In fact, every discrimination was conducted as the following procedure:

1. Choose 1 image from all the images as test data, and use the other images as training dataset.

2. Discriminate the test data between the class of "normal" and "abnormal".

3. Changing the test data, repeat from 1 to 2 until every image has been the test data.

Table 2 shows discrimination results for abnormal and normal cases respectively obtained by the three discrimination machines of LDA, NN, and L-SVM, where Precision and Recall represent the discrimination ratio. They are defined as

$$
\begin{gathered}
\text { Precision }=\frac{X_{h \cap c}}{X_{c}} \times 100 \\
\text { Recall }=\frac{X_{h \cap c}}{X_{h}} \times 100
\end{gathered}
$$

Where $X$ represents the number of images, $X_{h}$ is the number of the correct answers, and $X_{c}$ is the number of images discriminated by the proposed method. In addition, Figure 8 shows the discrimination ratios against change of the parameter $\partial$ in the Gaussian kernel used in N-SVM, where A and $\mathrm{N}$ means abnormal and normal cases, respectively. As shown in Table 2 and Figure 8, the discrimination ratios for each of normal and abnormal cases show more than $80 \%$ and there is no significant difference of the results between the users. Actually, in order to pass the reading test of mammography images in Japan, medical doctors have to correctly read at least $80 \%$ in each of cancer cases and normal cases. Hence, if the experimental results were evaluated from the clinical standpoint, the experimental results would show the proposed abnormalities are appropriately extracted for the discrimination of internal hemorrhoids caused by the congestion. Table 3 shows the mean value of each abnormality extracted from correct dataset of each case, where the number in the round blankets is the standard deviation.

Table 2: Experimental results

\begin{tabular}{|c|c|c|c|c|c|c|c|}
\hline \multirow{2}{*}{ User } & \multirow{2}{*}{ case } & \multicolumn{2}{|c|}{ LDA } & \multicolumn{2}{c|}{ NN } & \multicolumn{2}{c|}{ SVM } \\
\cline { 3 - 8 } & & Precision & Recall & Precision & Recall & Precision & Recall \\
\hline \multirow{2}{*}{ A } & normal & $85.4 \%$ & $78.1 \%$ & $83.3 \%$ & $82.5 \%$ & $80.5 \%$ & $80.6 \%$ \\
\cline { 2 - 8 } & abnormal & $78.7 \%$ & $85.9 \%$ & $84.3 \%$ & $85.0 \%$ & $81.5 \%$ & $87.1 \%$ \\
\hline \multirow{2}{*}{ B } & normal & $89.6 \%$ & $81.1 \%$ & $85.4 \%$ & $85.4 \%$ & $87.5 \%$ & $82.4 \%$ \\
\cline { 2 - 8 } & abnormal & $81.5 \%$ & $89.8 \%$ & $87.0 \%$ & $87.0 \%$ & $83.4 \%$ & $88.2 \%$ \\
\hline \multirow{2}{*}{ C } & normal & $84.4 \%$ & $72.3 \%$ & $86.5 \%$ & $83.8 \%$ & $87.5 \%$ & $80.0 \%$ \\
\cline { 2 - 8 } & abnormal & $71.3 \%$ & $83.7 \%$ & $85.2 \%$ & $87.6 \%$ & $80.6 \%$ & $87.9 \%$ \\
\hline
\end{tabular}




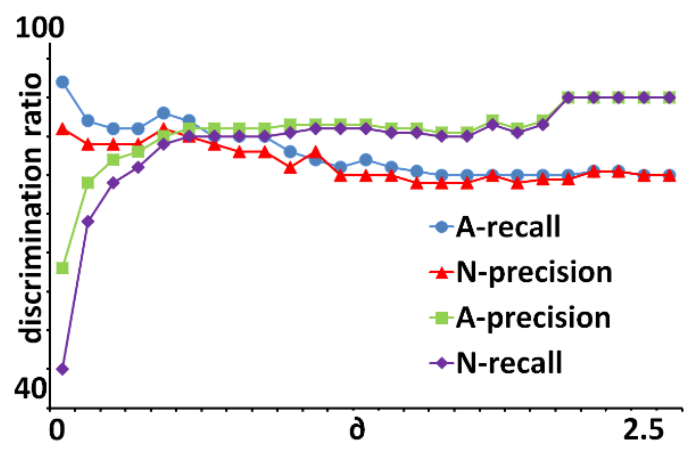

Figure 8: The discrimination ratio vs. $\partial$ of the Gaussian kernel in N-SVM

Table 3: Mean values of the abnormalities in each of correct abnormal and normal datasets.

(The number in the parenthesis is the standard deviation.)

\begin{tabular}{|c|c|c|c|c|}
\hline abnormality & $f_{1}$ & $f_{2}$ & $f_{3}$ & $f_{4}$ \\
\hline abnormal & $0.55(1.10)$ & $0.57(1.03)$ & $-0.55(0.80)$ & $0.59(0.99)$ \\
\hline normal & $-0.61(0.17)$ & $-0.64(0.39)$ & $0.62(0.81)$ & $-0.66(0.42)$ \\
\hline
\end{tabular}

Figure 9 shows an example of discrimination success for the abnormal case in all the discrimination machines. In Figure 9, (1) is the original image, (2) is the image where the tube has been removed from (1), (3) is the image converted by the stretch with saturation for (2), and (4) is the black and white image of congestive region extracted from (3), where the region is shown as the white color. In addition, Figure 10 shows an example of discrimination success for the normal case in all the discrimination machines, where (1), (2), (3) and (4) are the same as Figure 9, respectively.

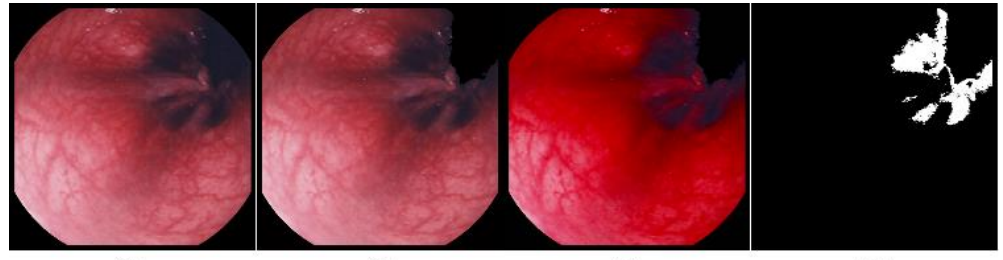

(1)

(3)

(4)

Figure 9: An abnormal case of discrimination success $(f 1=0.100, f 2=0.199, f 3=-0.741, f 4=0.174)$.

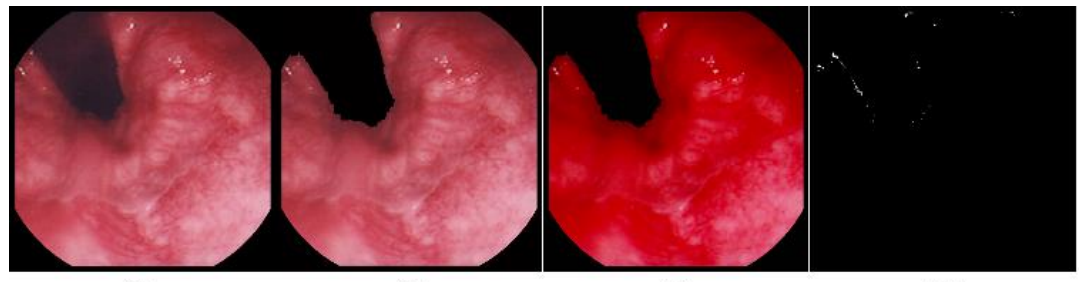

(1)

(2)

(3)

(4)

Figure 10: A normal case of discrimination success $\left(f_{1}=-0.766, f_{2}=-1.003, f_{3}=1.574, f_{4}=-1.273\right)$.

\section{Discussions}

In the experimental results, the number of images which were discrimination failure in all the discrimination machines was 15 , where 7 normal cases were diagnosed as abnormal cases and 8 abnormal cases were done as normal cases. Figure $11-14$ show examples of the discrimination failures, where (1), (2), (3) and (4) are the same as Figure 9, respectively. 
Koji Abe, Hidenori Takagi, Masahide Minami and Haiyan Tian; Computer-aided Diagnosis for Internal Hemorrhoids by Measuring the Congestive Extent in Endoscopic Images, Journal of Biomedical Engineering and Medical Imaging, Volume 1, No 6, Dec (2014) , pp 10-21

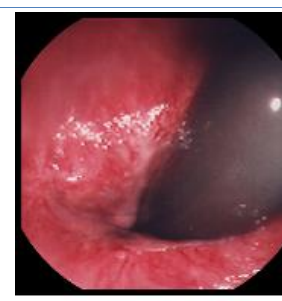

(1)

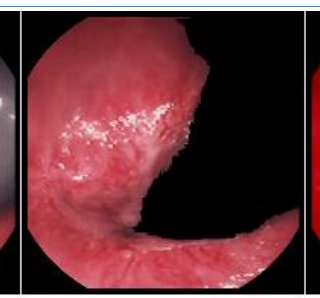

(2)

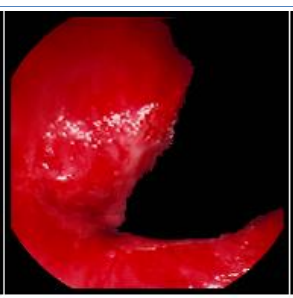

(3)

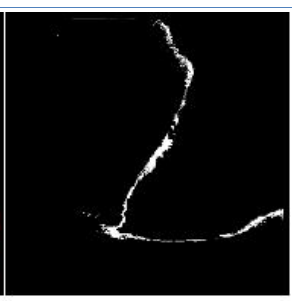

(4)

Figure 11: Failure case 1 (by the proposal: abnormal, correct answer: normal)

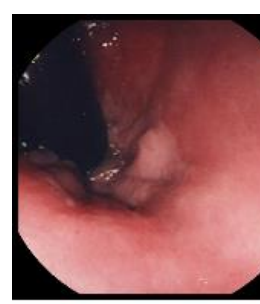

(1)

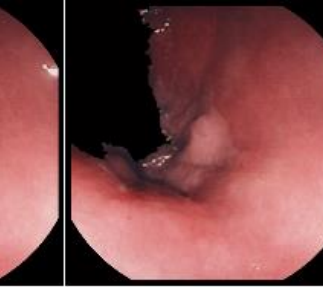

(2)

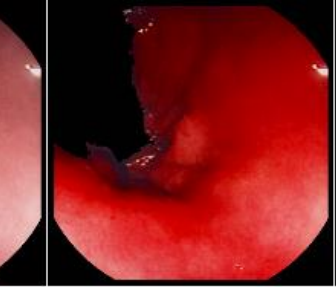

(3)

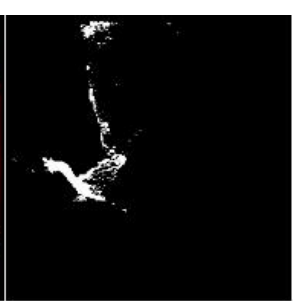

(4)

Figure 12: Failure case 2 (by the proposal: abnormal, correct answer: normal)

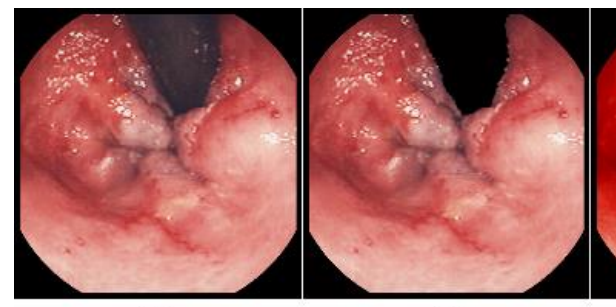

(1)
(2)

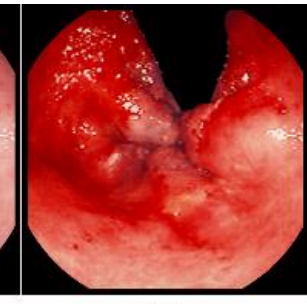

(3)

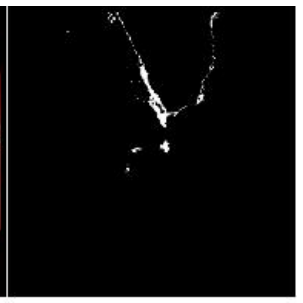

(4)

Figure 13: Failure case 3 (by the proposal: normal, correct answer: abnormal)

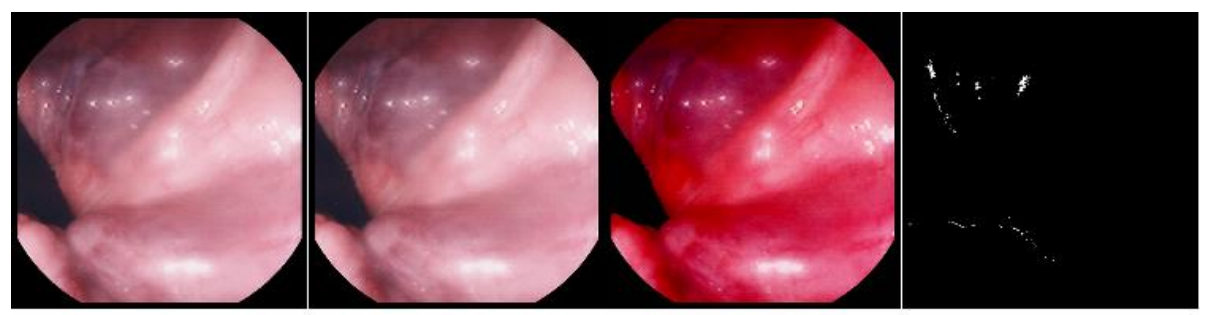

(1)

(2)

(3)

(4)

Figure 14: Failure case 4 (by the proposal: normal, correct answer: abnormal)

The normal cases which were diagnosed as abnormal cases can be divided broadly into two kinds. Figure 11 and Figure 12 show a case of each kind. In the case of Figure 11, we could confirm there is the congestive region though this case is a normal case, besides $f 1=-0.226$ and $f 2=0.446$, i.e., the features are closer to the mean value of them in abnormal case shown in Table 3. Actually, this case was internal hemorrhoids before and it is going to the recovery in progress; and a diagnostician concluded this case has already gone out of abnormal cases. The other 3 normal cases were the same as this. In the case of Figure 12, we can confirm that there is shade such as shadow appeared like a tunnel. Since the proposed method cannot distinguish between the congestive region and the shade, the proposed method cannot extract the features precisely in this kind of cases when deep shade is appeared around the tube. In fact, $\mathrm{f} 3$ and $\mathrm{f} 4$ are -0.301 and 0.067 , respectively, and they are closer to the mean values of them in abnormal 
case than normal case shown in Table 3. The other 2 normal cases were the same as this. In the future, it is necessary to consider a method for recognizing bumpy surface and its color in the endoscopic images.

Next, the abnormal cases which were diagnosed as normal cases can be also divided broadly into two kinds. Figure 13 and Figure 14 show a case of each kind. In the case of Figure 13, we can confirm there are some projections and swellings. Since the proposed abnormalities are designed by considering only the congestion, the abnormalities cannot apply to these symptoms. Regarding the image shown in Figure 13 , the abnormalities are $f_{1}=-0.633, f_{2}=-0.669, f_{3}=0.412$, and $f_{4}=-0.486$, respectively, and all of them are closer to the mean values of normal cases shown in Table 3. The other 3 abnormal cases were the same as this. In the future, it is necessary to consider a method for recognizing the projection and swelling parts in order to diagnose these symptoms separately. In the case of Figure 14, due to the lightning of the endoscope, change of hue has been occurred hence the abnormal case was discriminated as a normal case. The other 3 abnormal cases were the same as this. Regarding the image shown in Figure 14, the abnormalities are $f_{1}=-0.755, f_{2}=-1.018, f_{3}=0.885$, and $f_{4}=-0.208$, respectively, and all of them are closer to the mean values of normal cases shown in Table 3 . The lightning sometimes brings halation or change of hue into endoscopic images. If the location of the light source could be obtained, there could be possibility that the color of the image is reproduced into the original color. However, since this problem can also occur in other general images and it is still not solved, in order to cope with this case, it might be necessary to consider preparing a manual on taking desirable pictures for diagnosticians.

\section{Conclusions}

Aiming at showing a second opinion to diagnosticians and supporting non-experts in internal hemorrhoids, this paper has presented abnormalities for measuring the congestive extent in endoscopic images of internal hemorrhoids. The proposed abnormalities provide degree of congestive color density and size of the congestive region as features besides they could be used for a computer-aided diagnosis of internal hemorrhoids. Regarding the abnormalities as variables for discrimination of internal hemorrhoids, this paper has examined performance of the abnormalities by discriminating between normal and abnormal cases with discrimination machines. Experimental results of the discriminant trials have shown that the discrimination ratios for the proposed method have become more than $80 \%$ in almost of the trials. In addition, we have confirmed the proposed method could extract the congestive region well. However, the proposed method cannot still cope with abnormal cases caused by the other symptoms. And also, it is difficult to diagnose internal hemorrhoids to the images where hue have been changed due to the light of the endoscope.

Therefore, as future works, it is necessary to consider recognizing bumpy surface and its color in the images, the projection and swelling parts, and designing a manual on taking the desirable pictures for diagnosticians.

\section{REFERENCES}

[1]. B. Li and M.Q. Meng (2009), 'Computer-Aided Detection of Bleeding Regions for Capsule Endoscopy Images', IEEE Trans. Biomed. Eng., 56(4), 1032-1039. 
Koji Abe, Hidenori Takagi, Masahide Minami and Haiyan Tian; Computer-aided Diagnosis for Internal Hemorrhoids by Measuring the Congestive Extent in Endoscopic Images, Journal of Biomedical Engineering and Medical Imaging, Volume 1, No 6, Dec (2014) , pp 10-21

[2]. K-B Kim, S. Kim, and G-H Kim (2006), 'Analysis System of Endoscopic Image of Early Gastric Cancer', IEICE Trans. Fundam. Electron. Commun. Comput Sci., E89-A(10), 2662-2669.

[3]. M.P. Tjoa, S.M. Krishinan, C Kugean, P. Wang, and R. Doraiswami (2001), 'Segmentation of Clinical Endoscopic Image Based on Homogeneity and Hue', Proc. Annual Int. Conf. IEEE Eng. Med. Biol. Soc., 3, 2665-2668.

[4]. M. Xiao, S. Xia, and S.Wang (2005), 'Geometric Active Contour Model with Color and Intensity Priors for Medical Image Segmentation', Proc. 27th Annual Int. Conf. IEEE Eng. Meg. Biol. Soc., 6496-6499.

[5]. H. Tian, T. Srikanthan, and K. V. Asari (2001), 'Automatic segmentation slgorithm for the lumen region and boundary from endoscopic images', Med. Biol. Eng. Comput., 39(1), 8-14.

[6]. F. Vilarino, P. Spyridonos, F. Deiorio, J. Vitria, F. Azpiroz, and P. Radeva (2010), 'Intestinal Motility Assessment With Video Capsule Endoscopy : Autmatic of Phasic INtestial Contractions', IEEE Trans Med Imaging, 29(2), 246-259.

[7]. Y. Li, J. Sun, C-K Tang, and H-Y Shum (2004), 'Lazy Snapping' , ACM Trans. on Graphics, 23(3), 303-308.

[8]. M. Stone (1974), 'Cross-Validatory Choice and Assessment of Statistical Predictions', J. of the Royal Statistical Society, Series B (Methodological), 36(2), 111-147. 